\title{
入侵植物薇甘菊种群的遗传分化
}

\author{
李钧敏 ${ }^{13}$ 董 鸣 ${ }^{2 *}$ 钟章成 ${ }^{1}$ \\ (1 西南大学国家教育部三峡库区生态环境重点实验室, 重庆 400715) \\ （2 中国科学院植物研究所植被与环境变化国家重点实验室，北京１00093）（3 台州学院生态研究所，浙江临海 317000)
}

摘 要 利用简单重复序列区间 (Inter simple sequence repeat, ISSR)分子标记技术分析了入侵植物薇甘菊 (Mikania micrantha) 8 个种群的遗传多样性及遗传分化。12 个引物共扩增出 171 个位点 其中多态位点有 103 个, 多态位点百 分率 $(P \%)$ 为 $60.23 \%$ Shannon 信息指数 $(I)$ 为 0.2818 ,Nei 指数 $(h)$ 为 0.1849 薇甘菊在物种水平具有较高的遗传 多样性。AMOVA 显示薇甘菊具有较高的遗传分化 $36.49 \%$ 的变异发生在种群间 $63.51 \%$ 的变异发生于种群内 基 因分化系数 $\left(G_{\mathrm{ST}}\right)$ 为 0.3524 。种群间的基因流较高, 为 0.9187 。薇甘菊 8 个种群之间的遗传相似性很高, 平均为 0.9155 遗传距离很小, 平均为 0.088 。采用 UPGMA 法对 8 个种群进行聚类, 可以将 8 个种群分为两大类群, 即内 伶仃岛为一个类群, 而深圳与东莞内陆种群组成另一类群。薇甘菊现有遗传结构的形成与其生活史特性及入侵生 态学特性有关。

关键词 薇甘菊 入侵植物 遗传多样性 遗传分化 ISSR

\section{POPULATION GENETIC DIFFERENTIATIONS IN THE INVASIVE PLANT MIKANIA MICRANTHA IN CHINA}

\author{
LI Jun-Min ${ }^{13}$, DONG Ming ${ }^{2 *}$, and ZHONG Zhang-Cheng ${ }^{1}$ \\ ${ }^{I}$ The State Education Ministry's Key Laboratory for the Eco-environment of Three Gorges Reservoir Area , Southwest University , Chongqing 400715 , \\ China , ${ }^{2}$ Key Laboratory of Vegetation and Environmental Change , Institute of Botany , Chinese Academy of Sciences , Beijing 100093 , China , and \\ ${ }^{3}$ Institute of Ecology , Taizhou University , Linhai , Zhejiang 317000 , China
}

\begin{abstract}
Aims Genetic changes after introduction may contribute to the success of invasive plants. Our objective was to measure genetic diversity and genetic differentiation in the invasive Mikania micrantha in order to assess its invasive prospects.

Methods Inter-simple sequence repeat (ISSR) markers were used to analyze genetic diversity and genetic differentiation in eight populations of $M$. micrantha .

Important findings The 12 ISSR primers used in this study produced 171 loci , of which 103 were polymorphic. At the species level, the percentage of polymorphic loci $(P)$ was $60.23 \%$, Shannon's information in$\operatorname{dex}(I) 0.2818$ and Nei's gene diversity $(h) 0.1849$, indicating high genetic diversity in $M$. micrantha. Analysis of molecular variance (AMOVA) revealed that a high proportion (36.49\%) of the total genetic variation was partitioned among populations, suggesting significant genetic differentiations among the eight populations of $M$. micrantha. Gene differentiation coefficient $\left(G_{\mathrm{ST}}\right)$ among populations was 0.3524 , and gene flow among populations was 0.9187 . Average genetic similarity of the eight populations was 0.9155 , while average genetic distance was 0.088 4. Using unweighted pair group method arithmetic average (UPGMA), the eight populations were clustered into two groups : the two populations on Neilingding Island were one group and the other six from mainland China were the other.
\end{abstract}

Key words Mikania micrantha, invasive plant, genetic diversity, genetic differentiation, ISSR

薇甘菊( Mikania micrantha)，又名小花䒤泽兰， 为菊科假泽兰属 (Mikania) 植物, 为多年生草质或稍 木质藤本, 具䒤生茎, 攀援并缠绕幼树、作物或其它 植物，使其死亡(孔国辉等 ,2000a)。薇甘菊原产中、 南美洲，目前广泛存在于热带非洲、热带亚洲、澳大
利亚及南太平洋岛屿以及亚热带许多国家和地区， 是世界热带、亚热带地区危害严重的杂草之一(肯启 杰等, 2000)。20世纪 80 年代初在深圳首次采集到 薇甘菊标本 (孔国辉等, $2000 \mathrm{~b}$ )。薇甘菊在中国大 陆主要分布于 $21^{\circ} \sim 24^{\circ} \mathrm{N}, 111^{\circ} \sim 117^{\circ} \mathrm{E}$, 主要集中在 
广东省深圳市、东莞市、珠海市等地 (冯惠玲等, 2002)。目前, 国内外对薇甘菊的形态分类(孔国辉 等, 2000b; 王伯荪等, 2001)、生物学特性 (李鸣光 等, 2002)、生态学特性 (Swamy \& Ramakrishnan, 1987a ; 张炜银等,2002a，2002c ; 杨期和等,2003)、 分布和危害 (冯惠玲等, 2002) 、防除 (Swamy \& Ramakrishnan, 1987b; 邵婉婷等，2002；备启杰等， 2002 ; 邵华等，2002)、化学成分 (Nicollier \& Thompson , 1981 ; Bakir et al. ,2004; Wei et al.,2004)、化 感作用 (Ismail \& Chong, 2002) 等研究较多。作为一 种侵染性极强的有害杂草, 薇甘菊的分布范围较广， 生境条件多样，因此，从分子生态学的角度对不同侵 入地区及不同生态环境中的薇甘菊进行遗传多样性 分析 探索其遗传变异规律 对薇甘菊的控制和防除 有积极意义(张炜银等, 2002b)。

王伯荪等对薇甘菊叶绿体 trnL-trnF 内含子序列 进行分析 结果显示不同种群的薇甘菊的序列完全 相同 (王伯荪等，2004)。但曾国洋 ${ }^{1}$ 利用简单重复 序列区间 (Inter simple sequence repeat, ISSR)技术分 析了台湾岛 14 个薇甘菊种群的遗传多样性与遗传 分化, 认为台湾薇甘菊种群间有显著的遗传分化。 ISSR 技术是建立在 PCR 反应基础上的一种新的分 子生物学技术，由 Zietkiewicz 等(1994)于 1994 年创 建, 其基本原理是利用 $16 \sim 25 \mathrm{bp}$ 的加针简单重复 序列(Simple sequence repeat, SSR) 为引物, 对位于反 向排列的 SSR 之间的 DNA 序列进行 PCR 扩增( Ammiraju et al .,2001)。ISSR 引物序列较长, 扩增的是 重复序列之间的序列, 稳定性较高, 重复性较好, 可 以产生比 RAPD 多 3 5 倍的多态性 (Tsumura et al. , 1996 ; Hollingsworth et al., 1998 ; Esselman et al. , 1999)。ISSR 技术还具有 DNA 用量少、操作简单、实 验成本低等优点, 近年来已迅速应用于品种鉴定 (Ammiraju et al ，２001)，种质资源( Li \& Ge，2001) 和遗传多样性 (Wolfe et al. , 1998) 的研究, 并作为 构建遗传图谱的工具 (马翠兰等, 2001)。本研究采 用 ISSR 技术对广东省 8 个薇甘菊种群的遗传多样 性与遗传分化进行分析, 旨在揭示薇甘菊种群的遗 传多样性水平、种群间和种群内的遗传分化程度和 基因流，并据此分析其遗传结构的形成原因，从而预 测薇甘菊的潜在入侵趋势, 为薇甘菊的管理及防除 措施的确立提供基础理论依据。

\section{1 材料和方法}

\section{1 供试材料}

样品采集于广东省东莞市筌村区西湖乐园西平 水库边 $(\mathrm{XH})$ 、深圳市福田区民俗村南弃荒地 (MS)、 深圳市宝安区沙井镇大王山村水沟边 (DWS) 、深圳 市罗湖区大望村水库边 (DW)、梧桐山山坡上 (WT)、 去梧桐山的公路边 (GL) 和深圳市内伶仃岛山坡灌 丛上 (NLDI) 及相思林下 (NLDII) 共 8 个种群。每个 种群选取 20 株成年植体, 个体间相距 $30 \mathrm{~m}$ 以上, 并 确定植株之间无物理连接。采取植株的幼嫩叶片置 于保鲜袋中, 封口, 置于样品咜藏箱 (由超低温冰袋 保持冷藏条件)带回实验室, $-70{ }^{\circ} \mathrm{C}$ 低温冰箱保存, 供 DNA 提取。

\section{2 研究方法}

\subsubsection{DNA 提取和定量}

采用改进的 SDS 法 (李钧敏等，2002) 提取总 DNA。DNA 经 $0.8 \%$ 琼脂糖凝胶电泳分析, 用 GIS 凝 胶成像分析系统 (上海天能科技服务公司) 拍照定 量, 并稀释成终浓度为 $10 \mathrm{ng} \mu \mathrm{l}^{-1},-20{ }^{\circ} \mathrm{C}$ 保存备 用。

\subsubsection{ISSR 扩增及产物鉴定}

ISSR 引物是根据加拿大哥伦比亚大学 (University of British Columbia, Set No. 9 , No. 801-900) 公布 的序列, 由上海生工生物工程公司合成。扩增反应 在美国 Thermo 公司的 $\mathrm{P} \times 2$ 热循环仪中进行。经过 测试镁离子浓度、dNTP 浓度、模板 DNA 含量、引物 浓度、DNA 聚合酶量对 ISSR 反应结果的影响后, 确 定了最适的 ISSR 扩增反应条件为: $10 \mu \mathrm{l}$ PCR 反应

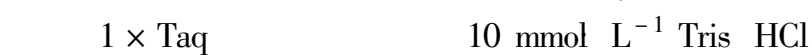
pH $9.0,50$ mmol $\mathrm{L}^{-1} \mathrm{KCl}, 0.1 \%$ Triton X-100) ,1.5 $\mathrm{mmol} \mathrm{L} \mathrm{L}^{-1} \mathrm{MgCl}_{2}, 1 \mathrm{U}$ Taq 酶(上海华美公司 ) ,20 ng 模板 DNA , 12 pmol 引物; ;ATP、dCTP、dGTP、dTTP 各 $0.15 \mathrm{mmol} \mathrm{L}^{-1}$ 。同时测试了 ISSR 扩增的最适退火 温度, 经优化的 PCR 扩增程序为: $94{ }^{\circ} \mathrm{C}$ 预变性 5 $\min , 94{ }^{\circ} \mathrm{C}$ 变性 $30 \mathrm{~s}, 49.3 \sim 60.6{ }^{\circ} \mathrm{C}$ 退火 $45 \mathrm{~s}$ (根据不 同引物确定不同退火温度),72 ${ }^{\circ} \mathrm{C}$ 延伸 $1.5 \mathrm{~min}$, 共 35 个循环, 72 ${ }^{\circ} \mathrm{C}$ 完全延伸 $5 \mathrm{~min}$ 。扩增产物在 $1.4 \%$ 的琼脂糖凝胶 (含 $0.5 \mu \mathrm{g} \mathrm{ml}{ }^{-1}$ 溴化乙锭) 中电泳, 电 泳缓冲液为 $0.5 \times \mathrm{TBE}$, 用 GIS 凝胶成像分析系 统 (上海天能科技服务公司) 拍照保存。用 $\lambda \mathrm{DNA} /$ 
$E c o R I+H i n d[\mid[$ 标准分子量参照物 (上海华美公司) 做 分子量标记。阴性对照加入除模板 DNA 外的以上各 成分, 用双蒸水代替总 DNA。从 100 个引物中篮选出 在 8 个薇甘菊种群中均可扩增出清晰条带, 且条带不 弥散，不模糊，重复性好，同时阴性对照中无带的 12 个引物作为正式扩增的 ISSR 扩增引物(表 1)。

表 1 ISSR 分析用的 12 个引物序列

Table 1 Sequences of 12 primers used in ISSR analysis

\begin{tabular}{clcl}
\hline $\begin{array}{c}\text { 引物 } \\
\text { Primers }\end{array}$ & \multicolumn{1}{c}{$\begin{array}{c}\text { 序列 } \\
\text { Sequences }\end{array}$} & $\begin{array}{c}\text { 引物 } \\
\text { Primers }\end{array}$ & $\begin{array}{c}\text { 序列 } \\
\text { Sequences }\end{array}$ \\
\hline UBC808 & $(\mathrm{AG})_{8} \mathrm{C}$ & $\mathrm{UBC} 873$ & $(\mathrm{GACA})_{4}$ \\
$\mathrm{UBC} 811$ & $(\mathrm{GA})_{8} \mathrm{C}$ & $\mathrm{UBC} 880$ & $(\mathrm{GGAGA})_{3}$ \\
$\mathrm{UBC} 841$ & $(\mathrm{GA})_{8} \mathrm{YC}$ & $\mathrm{UBC} 884$ & $\mathrm{HBH}(\mathrm{AG})_{7}$ \\
$\mathrm{UBC} 857$ & $(\mathrm{AC})_{8} \mathrm{YG}$ & $\mathrm{UBC} 885$ & $\mathrm{BHB}(\mathrm{GA})_{7}$ \\
$\mathrm{UBC} 864$ & $(\mathrm{ATG})_{6}$ & $\mathrm{UBC} 886$ & $\mathrm{VDV}(\mathrm{CT})_{7}$ \\
$\mathrm{UBC} 868$ & $(\mathrm{GAA})_{6}$ & $\mathrm{UBC} 887$ & $\mathrm{DVD}(\mathrm{TC})_{7}$ \\
\hline
\end{tabular}

\subsection{3 数据统计与分析}

对照反应产物在凝胶上的对应位置, 有带记为 1 ” 无带记为 0 ”, 得到 ISSR 分析的原始数据矩阵。 采用 POPGEN32 软件 ( Yeh \& Boyle, 1997) 计算多态 位点百分率 $(P \%)$ 、Shannon 信息指数 $(I)$ 和 Nei 指数 ( $h$ )用来估算基因多样性。采用 DCFA 1.1 软件(张 富民和葛颂，2002)计算欧氏距离平方和，建立遗传 距离矩阵, 并利用 WINAMOVA 1.5 软件 (Excoffier et $a l$ ，1992) 进行分子方差分析。利用 AMOVA 计算 种群内、种群间的变异方差分布。另外 根据基因频 率矩阵 利用 POPGEN32 软件计算了总种群基因多 样度 $\left(H_{\mathrm{t}}\right)$ ，各种群基因多样度 $\left(H_{\mathrm{s}}\right)$ 、遗传分化系数 $\left(G_{\mathrm{ST}}\right)$ 和基因流。利用 Shannon 信息指数计算基于 各引物扩增条带在种群 $\left(H_{\mathrm{pop}}\right)$ 和物种水平的表型多 样性 $\left(H_{\mathrm{sp}}\right)$ ，分别根据 $\left(H_{\mathrm{pop}} / H_{\mathrm{sp}}\right)$ 和 $\left(H_{\mathrm{sp}}-H_{\mathrm{pop}}\right) / H_{\mathrm{sp}}$ 计算种群内和种群间变异所占的比例。为了分析种 群间的遗传关系, 计算了 Nei 无偏差遗传距离 (Nei , 1972) 和遗传相似度。应用 XLSTAT-Pro 2007 软件 (http :/ /www. kovcomp. co. uk/xlstat/index. html) 进行 Mantel 检测 检测遗传距离和地理距离之间是否存 在相关性。根据种群间的遗传距离, 采用算术平均 数的非加权成组配对法 (Unweighted pair group method with arithmetic mean, UPGMA) 对各种群进行聚类分 析。

\section{2 结果与分析}

2.1 遗传多样性
利用 12 个 ISSR 引物对 8 个薇甘菊种群共 160 个个体的 DNA 样品进行了 ISSR 分析, 每个引物均 能扩增出清晰的可重复的条带,其中引物 UBC811 对 GL 种群的 20 个个体 DNA 样品扩增结果见图 1。 12 个引物共扩增出 171 条带, 平均每个引物有 14.25 个条带 其中有 103 个条带为多态条带, 占 $60.23 \%$ 。 各种群的多态位点百分率 $(P \%)$ 的变化范围在 $32.16 \% \sim 40.35 \%$ 之间（表 2)，平均为 $34.58 \%$,以 XH种群最高,NLD I 种群和 GL 种群相同,均为最 低。薇甘菊各种群的 Shannon 信息指数 $(I)$ 的变化 范围在 $0.1522 \sim 0.2335$ 之间, 平均为 0.1789 ,物种 水平为 0.2818 (表 2)。各种群的 $\mathrm{Nei}$ 基因多样性 $(h)$ 的变化范围在 $0.0997 \sim 0.1598$ 之间, 平均为 0.1197 , 物种水平为 0.1849 。

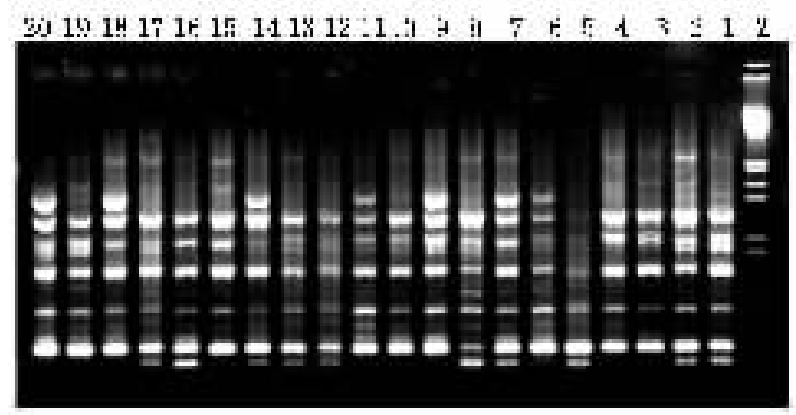

图 1 薇甘菊去梧桐山公路边 (GL)种群引物 UBC 811 扩增结果

Fig. 1 ISSR amplification of Mikania micrantha in GL population generated with primer UBC 811

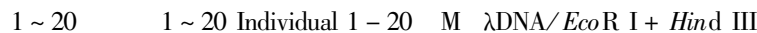
分子量参照物 $\lambda \mathrm{DNA} / E c o \mathrm{R} \mathrm{I}+$ Hind III standard molecular weight marker

\section{2 种群间的遗传分化}

AMOVA 分子变异分析显示在总的遗传变异中, $36.49 \%$ 的变异发生在种群间, 63.51\%的变异发生 于种群内 (表 3 ), 种群间有极显著的遗传分化 $\left(\Phi_{\mathrm{ST}}=0.365, p<0.001\right)$ 。根据 $I$ 计算的种群内和 种群间的遗传变异如表 5 所示,在总的遗传多样性 中，种群内的遗传多样性占 $65.61 \%$, 种群间占 $34.39 \%$ 。由 $h$ 计算的种群间基因分化系数 $\left(G_{\mathrm{ST}}\right)$ 为 0.3524 (表 4)。这些特征都反映出薇甘菊大部分遗 传变异存在于种群内, 小部分变异存在于种群间。 由 $G_{\mathrm{ST}}$ 所估算的基因流为 0.9187 (表 4) ,表明薇甘 菊具有较高的基因流。

\section{3 遗传距离}

利用 POPGENE 软件计算的结果可知 (表 5),8 个种群平均遗传相似度为 0.9155 , 平均遗传距离为 0.0884 。 WT 和 GL之间的遗传距离最小, 为 0.0513 ;NLD I 和 GL 种群之间的遗传距离最大, 为 0.2304 .8 个薇甘菊种群间的地理距离如表 6 所示。 
表 2 薇甘菊种群的遗传多样性

Table 2 Estimates of genetic diversity within populations of Mikania micrantha

\begin{tabular}{lcccc}
\hline $\begin{array}{c}\text { 种群(代码) } \\
\text { Population(Code) }\end{array}$ & $\begin{array}{c}\text { 多态位点数 } \\
\text { Number of polymorphic } \\
\text { loci }\end{array}$ & $\begin{array}{c}\text { 多态位点百分率 }(\%) \\
\text { Percetage of polymorphic } \\
\text { loci }(P)\end{array}$ & $\begin{array}{c}\text { Shannon 信息指数 } \\
\text { Shannon' s information index } \\
(I)\end{array}$ & $\begin{array}{c}\text { Nei 基因多样性 } \\
\text { Nei' s genet diversity } \\
(h)\end{array}$ \\
\hline 西湖乐园 (XH) & 69 & 40.35 & 0.2335 & 0.1598 \\
民俗村 (MS) & 59 & 34.50 & 0.1791 & 0.1189 \\
大王山 (DWS) & 62 & 36.26 & 0.1912 & 0.1295 \\
大望村 (DW) & 58 & 33.92 & 0.1901 & 0.1284 \\
内伶仃岛 I (NLD I) & 55 & 32.16 & 0.1552 & 0.1026 \\
内伶仃岛 II (NLD II) & 57 & 33.33 & 0.1658 & 0.1108 \\
梧桐山 (WT) & 58 & 33.92 & 0.1640 & 0.1082 \\
去梧桐山公路边 (GL) & 55 & 32.16 & 0.1522 & 0.0997 \\
种 Speices & 103 & 60.23 & 0.2818 & 0.1849 \\
\hline
\end{tabular}

每个种群的取样数均为 20 The sample size of every population is 20 individuals XH : Xihu amusement park MS : Ethnic village DWS : Dawangshan mountain DW : Dawang village NLD I : Neilingding island I NLD II : Neilingding island II WT : Wutong mountain GL : Road to ward wutong mountain

表 38 个薇甘菊种群的 AMOVA 分析

Table 3 Analysis of molecular variance (AMOVA) for the eight populations of Mikania micrantha

\begin{tabular}{ccccc}
\hline $\begin{array}{c}\text { 变异来源 } \\
\text { Source of variance }\end{array}$ & $\begin{array}{c}\text { 自由度 } \\
d f\end{array}$ & $\begin{array}{c}\text { 变异组分 } \\
\text { Variance component }\end{array}$ & $\begin{array}{c}\text { 百分率 } \\
\text { Pcentage }\end{array}$ & $\begin{array}{c}p \text { 值 } \\
\text { value }\end{array}$ \\
\hline 种群间 Among popualtions & 7 & 5.2577 & 36.49 & $<0.001$ \\
种群内 Within populations & 152 & 9.1507 & 63.51 & $<0.001$ \\
\hline
\end{tabular}

* : 1000 次交换 No. of permutation $=1000$

表 48 个薇甘菊种群的遗传分化

Table 4 Genetic differentiations within and among the eight populations of Mikania micrantha

\begin{tabular}{|c|c|c|c|}
\hline Shannon 信息指数 Shannon's information index & \multicolumn{3}{|c|}{ Nei 基因多样性 Nei's gene diversity } \\
\hline $\begin{array}{l}\text { 种群内遗传多样性 } \\
\text { Within population genetic diversity, } H_{\text {pop }}(S D)\end{array}$ & $\begin{array}{c}0.1849 \\
(0.0378)\end{array}$ & $\begin{array}{l}\text { 种群内基因多样性 } \\
\text { Within population gene diversity , } H_{\mathrm{S}}(S D)\end{array}$ & $\begin{array}{c}0.1197 \\
(0.0174)\end{array}$ \\
\hline $\begin{array}{l}\text { 种群总的遗传多样性 } \\
\text { Total genetic diversity }, H_{\mathrm{sp}}(S D)\end{array}$ & $\begin{array}{c}0.2818 \\
(0.2753)\end{array}$ & $\begin{array}{l}\text { 种群总的基因多样性 } \\
\text { Total gene diversity }, H_{\mathrm{T}}(S D)\end{array}$ & $\begin{array}{c}0.1849 \\
(0.1943)\end{array}$ \\
\hline $\begin{array}{l}\text { 种群内遗传多样性比率 } \\
\text { Ratio of genetic diversity within population, } H_{\mathrm{pop}} / H_{\mathrm{sp}}\end{array}$ & 0.6561 & $\begin{array}{l}\text { 种群内基因多样性比率 } \\
\text { Ratio of gene diversity within population, } H_{\mathrm{s}} / H_{\mathrm{T}}\end{array}$ & 0.6476 \\
\hline $\begin{array}{l}\text { 种群间遗传多样性比率 } \\
\text { Ratio of genetic diversity among population, }\left(H_{\mathrm{sp}}-H_{\mathrm{pop}}\right) / H_{\mathrm{sp}}\end{array}$ & 0.3439 & $\begin{array}{l}\text { 遗传分化系数 } \\
\text { Gene differentiation among populations, } G_{\mathrm{ST}}\end{array}$ & 0.3524 \\
\hline & & $\begin{array}{l}\text { 基因流 } \\
\text { Gene flow }, N_{\mathrm{m}}\end{array}$ & 0.9187 \\
\hline
\end{tabular}

经 Mantel 检验, Nei's 遗传距离与地理距离之间不存 在显著性相关 $(r=0.287, p=0.141)$ 。

采用 UPGMA 法对 8 个种群进行聚类(图 2) ,大 致可以将 8 个种群分为两大类群, 即内伶仃岛为一 个类群, 而深圳与东莞内陆种群组成另一类群。在 内陆类群中 地理距离较近的种群先聚在一起 , 如深 圳市 3 个地理距离最近的 WT、GL 和 DW 种群先聚 成一组, 而地理距离较近的 XH 和 DWS 种群聚成另 一组 然后两组聚在一起再与 MS 种群聚成一个类 群。

\section{3 讨 论}

外来入侵物种经常是起源于少数几个个体, 由 于遗传的瓶颈效应和建立者效应常具有较低的遗传 多样性(Nei et al ，1975)，如 Novak 和 Mack (1993) 利用等位酶技术检测到早雀麦 (Bromus tectorum) 的 $P \%$ 仅为 $3.46 \% ; \mathrm{Ye}$ 等 (2004) 利用 ISSR 标记检测 到中国入侵种香泽兰 (Chromolaena odorata) 的 $P \%$ 仅为 $2.35 \%$; Ren 等 (2005) 利用 RAPD 标记检测到 中国入侵种水葫芦 (Eichhornia crassipes) 的 $P \%$ 为 
表 58 个薇甘菊种群间的遗传相似度与遗传距离

Table 5 Genetic similarity and genetic distances among the eight populations of Mikania micrantha

\begin{tabular}{lcccccccc}
\hline \multicolumn{1}{c}{ 种群 Population } & XH & MS & DWS & DW & NLD I & NLD II & WT & GL \\
\hline 西湖乐园 (XH) & - & 0.9122 & 0.9279 & 0.9168 & 0.9139 & 0.9046 & 0.9285 & 0.9133 \\
民俗村( MS) & 0.0919 & - & 0.9002 & 0.9204 & 0.9025 & 0.8942 & 0.9257 & 0.9003 \\
大王山(DWS) & 0.0748 & 0.1051 & - & 0.9247 & 0.9062 & 0.9048 & 0.9285 & 0.8954 \\
大望村(DW) & 0.0869 & 0.0830 & 0.0783 & - & 0.9228 & 0.9112 & 0.9339 & 0.9153 \\
内伶仃岛 I (NLD I) & 0.0901 & 0.1026 & 0.0985 & 0.0804 & - & 0.9431 & 0.9209 & 0.893 \\
内伶仃岛 II (NLD II) & 0.1003 & 0.1118 & 0.1000 & 0.0930 & 0.0585 & - & 0.9309 & 0.8935 \\
梧桐山(WT) & 0.0742 & 0.0772 & 0.0742 & 0.0684 & 0.0824 & 0.0716 & - & 0.9500 \\
去梧桐山公路边 (GL) & 0.0906 & 0.1050 & 0.1104 & 0.0885 & 0.1132 & 0.1126 & 0.0513 & - \\
\hline
\end{tabular}

对角线上方为 Nei 遗传相似度，对角线下方为遗传距离 Nei's genetic similarity (above diagonal) and Nei' s genetic distances (below diagonal) XH、MS、DWS、DW、NLD I、NLD II、WT、GL : 同表 2 See Table 2

表 68 个薇甘菊种群间的地理距离 $(\mathbf{k m})$

Table 6 Geographical distances $(\mathrm{km})$ among the eight populations of Mikania micrantha

\begin{tabular}{|c|c|c|c|c|c|c|c|c|}
\hline 种群 Population & $\mathrm{XH}$ & MS & DWS & DW & NLD I & NLD II & WT & GL \\
\hline 西湖乐园 (XH) & - & & & & & & & \\
\hline 民俗村 (MS) & 50.3 & - & & & & & & \\
\hline 大王山(DWS) & 26 & 26.3 & - & & & & & \\
\hline 大望村 (DW) & 54.7 & 19 & 37.6 & - & & & & \\
\hline 内伶仃岛 I (NLD I) & 103.9 & 22.1 & 29.1 & 40.5 & - & & & \\
\hline 内伶仃岛 II (NLD II) & 104.2 & 22.5 & 29.6 & 40.7 & 1.6 & - & & \\
\hline 梧桐山(WT) & 54.2 & 19.5 & 37.2 & 0.6 & 41.1 & 41.3 & - & \\
\hline 去梧桐山公路边 (GL) & 54.4 & 19.2 & 37.4 & 0.3 & 40.9 & 41.1 & 0.3 & - \\
\hline
\end{tabular}

XH、MS、DWS、DW、NLD I、NLD II、WT、GL : 同表 2 See Table 2

遗传距离 Genetic distance

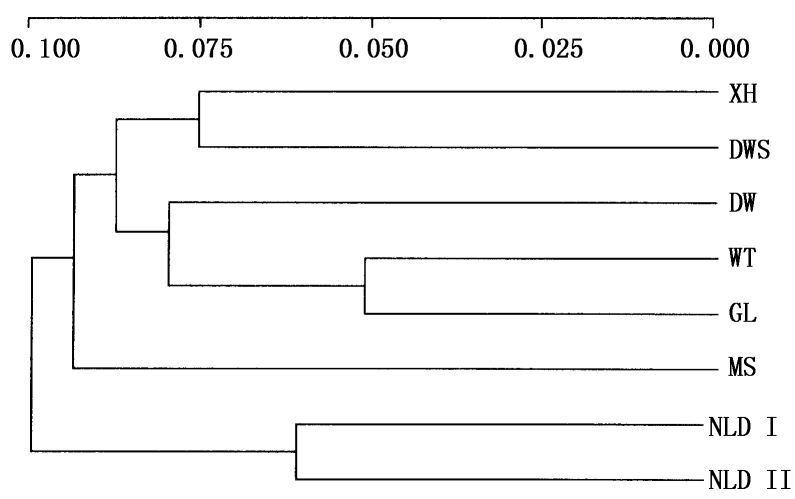

图 28 个薇甘菊种群间的遗传距离聚类图

Fig.2 Dengrogram produced by POPGENE software for the eight populations of Mikania micrantha

XH、MS、DWS、DW、NLD I、NLD II、WT、GL同表 2 See Table 2

$32 \%$ 。近年来, 随着分子标记技术的发展, 关于入侵 植物的遗传变异的研究报道越来越多。等位酶研究 显示外来的被子植物常具有较高的遗传变异 ( Hamrick et al . , 1979 , 1992 ; Hamrick \& Godt , 1990) 如美 国入侵种忍冬 ( Lonicera japonica ) ( $P \%=75 \%$; Schierenbeck et al ，1995)，北美入侵种香踠豆 (Lathyrus latifolius) ( $P \%=81 \%$; Godt \& Hamrick , 1991)。Meekins 等(2001)利用 ISSR 标记发现北美入
侵种葱芥 (Alliaria petiolata) 具有很高的遗传变异 $(P \%=91 \%)$, Li 等(2005) 也利用 ISSR 标记发现鸭 舌草 (Monochoria vaginalis) 具有较高的遗传变异 $(P \%=78.38 \%)$ 。本研究显示薇甘菊在物种水平 具有较高的遗传多样性 $(P \%=60.23 \%, I=$ $0.2818, h=0.1849)$ 。Pappert 等(2000)认为有两种 类型可能性可以导致入侵植物种群内高的遗传多样 性：一是现有杂草种群可能由多个不同来源的个体 所建立, 因此能保护较高的遗传多样性水平; 另外一 些杂草种群可能是由少数奠基者个体所建立, 但在 植物种群发育的阶段中, 由于花粉流、幼苗更新等可 以引入额外的基因多样性，从而导致种群具有高的 遗传多样性。薇甘菊种群间的基因流达到 0.9187 , 而短距离的种群间, 如 WT 种群、GL 种群和 DW 种 群之间的基因流则高达 1.447 。 结合薇甘菊的生 物学特性及强大的有性繁殖能力, 推测薇甘菊的高 遗传多样性可能是由于有性繁殖形成的高基因流、 高幼苗更新率所致。薇甘菊种群水平具有中等偏低 的遗传多样性,多态位点百分率在 $32.16 \%$ $40.35 \%$ 之间变动, 平均为 $34.58 \%$ 。薇甘菊各种群 的遗传多样性与其所适生的环境有关,杨期和等 (2003) 认为环境因素如土壤肥力、水分、生境均对薇 甘菊开花结实有明显的影响, 并发现塘泥等土壤中 
薇甘菊的结实率高, 而山地相对较低。XH、DW 和 DWS 种群生长于水库和水沟边, 土壤水肥充足, 结 实率高, 有性生殖效率高, 因此具有较高的遗传多样 性; 而 NLD I、NLD II、WT 和 GL 种群生长于山地, 有 性生殖效率较低, 因此具有较低的遗传多样性。

入侵植物常具有快速进化的能力，如通过遗传 漂变、建立者种群的快速进化 (Brown \& Marshall 1981)，入侵地种群的种内或种间杂交可能产生新的 基因型，对新环境的适应（Mooney \& Cleland 2001； Sakai et al. , 2001) 等等。入侵植物的遗传变异常大 部分存在于种群间, 小部分存在于种群内 (Brown \& Marshall，1981），这可能是由于自然选择、遗传漂 变、迁移和突变 (Wright, 1978)。对于入侵物种来 说, 低的种间遗传分化可能是由于遗传漂变、建立者 效应和环境变异的共同结果 (Brown \& Marshall, 1981)。而薇甘菊种群的遗传结构刚好与此相反。 AMOVA 分子变异分析显示在总的遗传变异中, $36.49 \%$ 的变异发生在种群间, 63. $51 \%$ 的变异发生 于种群内。曾国洋 ${ }^{1)}$ 利用 ISSR 技术分析台湾地区 14 个薇甘菊种群的遗传分化, AMOVA 分子变异分 析显示 $47.41 \%$ 的变异发生于种群间, 而 $52.59 \%$ 的 变异发生于种群内。薇甘菊兼有有性生殖与无性生 殖 薇甘菊的遗传结构的形成可能与其生活史特性 有关。薇甘菊营养体的茎节处可以生根, 伸入土壤 吸取养分, 进行营养繁殖, 为典型的克隆植物, 一旦 定居下来,一株植株在几个月内能够覆盖大片面积 （温达志等，2000）;但薇甘菊又进行有性生殖, 薇甘 菊开花结实量大, 花的生物量可占地上部分总生物 量的 $38.7 \% \sim 42.8 \%$, 薇甘菊种子小且轻, 具冠毛, 成熟后随风漂散(胡玉佳和毕培曦, 1994)。Govindaraju（1988)指出风媒植物常具有较高的遗传变异。 Hamrick 和 Godt (1989) 指出采用等位酶标记分析兼 有有性与无性繁殖的物种的 $G_{\mathrm{ST}}$ 平均为 0.213 。Pappert 等 (2000)利用等位酶标记发现美洲东南部的入 侵植物野葛 ( Pueraria lobata) 的大部分变异也存在于 种群内, 而小部分变异存在于种群间 $\left(G_{\mathrm{ST}}=0.199\right)$ 。 Aguinagalde 等(2005)指出采用 RAPD 标记分析兼有 有性与无性繁殖的欧洲温带物种的 $G_{\mathrm{ST}}$ 平均为 0.380 。本研究采用 ISSR 标记分析薇甘菊种群间 $G_{\mathrm{ST}}$ 为 0.3524 ,与此数据相似。

\section{入侵物种的遗传变异可用来确定入侵物种的地}

理起源,也可确定是否是单个或多个种群起源 (Meekins et al.,2001)。薇甘菊 8 个种群具有较高 的遗传相似度, 平均为 0.9155 较小的遗传距离, 平 均为 0.088 。 经 Mantel 检验, Nei' $\mathrm{s}$ 遗传距离与地 理距离之间不存在显著性相关 $(r=0.287, p=$ $0.141)$, 这与曾国洋 ${ }^{1)}$ 所揭示的台湾岛上薇甘菊种群 的结果相一致。采用 UPGMA 法对 8 个种群进行聚 类, 可以将 8 个种群分为两大类群, 即内伶仃岛为一 个类群, 而深圳与东莞内陆种群组成另一个种群。 薇甘菊远距离传播是借助于人的力量, 如货物运输 和交通贸易等完成的，但是短距离的传播扩散则是 由其自身的瘦果扩散能力，加之风力来完成的(张炜 银等 2002b)。因此, 推测薇甘菊入侵的主要途径可 能为人为扩散和自然扩散，如 XH、NLD、DWS、MS、 WT 种群均为人为因素造成的不同的入侵起源, 而 WT、GL 和 DW 种群之间, NLD I 和 NLD II 种群之间 则可能是由一个入侵种群经过有性生殖形成的大量 种子,再自然扩散而形成。

入侵植物遗传多样性及遗传分化可用于指导生 物控制媒介的篮选, 可以确定生物防治的敏感种群 (Nissen et al. , 1995)。如北美的两种不同生态型的 空心莲子草 (Alternanthera philoxeroides), 在遗传上是 不同的，对于生物控制媒介的敏感性也不同（Wain et al . , 1984)。对于克隆的入侵植物来说, 无性繁殖 导致物种的遗传一致度较高，生物控制媒介很容易 适应宿主的基因型，易于被天敌控制（Ye et al．， 2003)。而薇甘菊同时兼有有性生殖与无性生殖, 具 有较高的遗传多样性, 缺乏有效的生物控制媒介。 不同入侵地的薇甘菊种群可能具有不同的起源, 因 此对薇甘菊的防治要多个地区同时进行以提高生物 防治的效率。另外, 具有较高遗传变异的入侵物种 的种群与生物控制媒介之间易发生抗性进化，导致 防治难度增加(Brown \& Marshall，1981)。因此，对薇 甘菊开展生物防治时, 要针对其遗传多样性与遗传 变异进行深入探讨。

\section{参 考 文 献}

Aguinagalde I, Hampe A, Mohanty A, Martín JP, Duminil J, Petit RJ (2005). Effects of life-history traits and species distribution on genetic structure at maternally inherited markers in European trees and shrubs. Journal of Biogeography, 32, 329-339.

Ammiraju JSS, Dholakin BB, Sntra DK, Singh H, Lagu MD,

1) Zeng GY(曾国洋) (2003). Population genetic variation of Mikania species in Taiwan(台湾蔓泽兰属植物之族群遗传变异). MD dissertation , Sun Yat-Sen Univesrity , Taiwan. (in Chinese) 
Tamhankar SA, Dhaiual HS, Rao VS, Gupta VS, Ranjekar PK (2001). Identification of inter simple sequence repeat (ISSR) markers associated with seed size in wheat. Theoretical Application of Genetics, 102, 726 - 732 .

Bakir M, Facey PC, Hassan I, Mulder WH, Porter RB (2004). Mikanolide from Jamaican Mikania micrantha. Acta Crystallographica, Section C, Crystal Structure Communications, 60, $798-800$

Brown AHD, Marshall DR (1981). Evolutionary changes accompanying colonization in plants. In: Scudder GCE, Reveal JL eds. Colonization, Succession and Stability. Blackwell Scientific Publications, Oxford.

Esselman EJ, Jianqiang L, Crawford DJ, Windus JL, Wolfe AD (1999). Clonal diversity in the rare Calamagrostis porteri spp. insperata (Poaceae) : comparative results for allozymes and random amplified polymorphic DNA (RAPD) and intersimple sequence repeat (ISSR) markers. Molecular Ecology, 8, 443 451 .

Excoffier L, Smouse PF, Quattro JM (1992). Analysis of molecular variance inferred from metric distances among DNA haplotypes: application to human mitochondrial DNA restriction data. Genetics, 131, $479-491$.

Feng HL(冯惠玲), Cao HL(曹洪麟), Liang XD(梁晓东), Zhou $\mathrm{X}$ (周霞), $\mathrm{Ye} \mathrm{WH}$ (叶万辉) (2002). The distribution and harmful effect of Mikania micrantha in Guangdong. Journal of Tropical and Subtropical Botany (热带亚热带植物学报), 10, 263 - 270. (in Chinese with English abstract)

Godt MJW, Hamrick JL(1991) . Genetic variation in Lathyrus latifolius (Leguminosae). American Journal of Botany, 78, 1163 1171 .

Govindaraju DR (1988). Relationship between dispersal ability and levels of gene flow in plants. Oikos, 52, 31-35.

Hamrick JL, Godt MJW ( 1989 ). Allozyme diversity in plant species. In: Brown AHD, Clegg MT, Kahler AL, Weir BS eds. Plant Population Genetics, Breeding, and Genetic Resources. Sinauer, Sunderland, USA.

Hamrick JL, Godt MJW ( 1990 ). Allozyme diversity in plant species. In: Brown AHD, Clegg MT, Kahler AL, Weir BS eds. Plant Population Genetics, Breeding, and Genetic Resources. Sinauer, Sunderland, USA.

Hamrick JL, Godt MJW, Sherman-Broyles SL (1992) . Factors influencing levels of genetic diversity in woody plant species. New Forests, 6, $95-124$.

Hamrick JL, Linhart YB, Mitton JB (1979) . Relationships between life history characteristics and electrophoretically detectable genetic variation in plants. Annual Review of Ecological Systematics, 10,
$173-200$.

Hollingsworth ML, Hollingsworth PM, Jenkins GI, Bailey JP, Ferris C (1998). The use of molecular markers to study patterns of genotypic diversity in some invasive alien Fallopia spp. (Polygonaceae). Molecular Ecology, 7, 1681 - 1691.

Hu YJ (胡玉佳), Paul PHB (毕培曦) (1994). A study on life cycle and response to herbicides of Mikania micrantha. Acta Scientiarum Naturalium Universitatis Sunyatseni (中山大学学报 (自 然科学版) ), 33 (4), 88 - 95. (in Chinese with English abstract)

Ismail BS, Chong TV (2002). Effects of aqueous extracts and decomposition of Mikania micrantha H. B. K. debris on selected agronomic crops. Weed Biology and Management, 2, 31-38.

Kong GH(孔国辉), Wu QG (吴七根), Hu QM(胡启明), Ye WH(叶万辉) (2000a). Further supplementary data on Mikania micrantha H. B.K. (Asteraceae). Journal of Tropical and Subtropical Botany (热带亚热带植物学报)，8(2)，128-130. (in Chinese with English abstract)

Kong GH (孔国辉), Wu QG (吴七根), Hu QM (胡启明) (2000b) . Emergence of invasive weed Mikania micrantha H. B. K. in our county. Journal of Tropical and Subtropical Botany (热 带亚热带植物学报)，8(1)，27. (in Chinese)

$\operatorname{Li} \mathrm{JM}($ 李钧敏), Ke SS(柯世省), Jin ZX(金则新) (2002). Extraction and determination of DNA from Heptacodium miconioides. Guihaia (广西植物 ), 22, 499 - 502. (in Chinese with English abstract)

Li MG (李鸣光), Zhang WY (张炜银), Wang BS (王伯荪), Zhang JL(张军丽), Zan QJ (备启杰), Wang YJ (王勇军) (2002) . A preliminary study on the seed germination in Mikania micrantha. Acta Scientiarum Naturalium Universitis Sunyatseni (中山大学学报 (自然科学版) ), 41 (6), 57 - 59. (in Chinese with English abstract)

Li WG, Shen JJ, Wang JB (2005). Genetic diversity of the annual weed Monochoria vaginalis in southern China detected by random amplified polymorphic DNA and inter-simple sequence repeat analyses. Weed Research, 45, 424-430.

Li A, Ge S (2001). Genetic variation and colonal diversity of Psammochloa villosa (Poaceae) detected by ISSR markers. Annual of Botany, 87, $585-590$.

Ma CL(马翠兰), Liu XS(刘星释), Zhang YL(张玉兰) (2001). Application of DNA molecular markers to fruit trees. Journal of Inner Mongolia Agriculature University (Natural Science Edition) (内蒙古农业大学报 (自然科学版) ), 22(2), 105-112。（in Chinese with English abstract)

Meekins JF, Ballard HE, McCarthy BC (2001). Genetic variation and molecular biogeography of a North American invasive plant 
species (Alliaria Petiolata, Brassicaceae). International Journal of Plant Science, 162, 161 - 169.

Mooney HA, Cleland EE (2001). The evolutionary impact of invasive species. Proceedings of the National Academy Sciences of the United States of America, 98, 5446 - 5451

Nei M (1972). Genetic distance between populations. American Naturalist, 106, $283-292$.

Nei M, Maruyama T, Chakraborty R (1975). The bottleneck effect and genetic variability in populations. Evolution, 29, $1-10$.

Nicollier G, Thompson AC (1981). Essential oil and terpenoids of Mikania micrantha. Phytochemistry, 20, 2587 - 2588.

Nissen SJ, Masters RA, Lee DJ, Rowe ML (1995). DNA-based marker systems to determine genetic diversity of weedy species and their application to biocontrol. Weed Science, 43, $504-513$.

Novak SJ, Mack RN (1993). Genetic variation in Bromus tectorum (Poaceae): comparison between native and introduced populations. Heredity, 71, $167-176$.

Pappert RA, Hamrick JL, Donovan LA (2000). Genetic variation in Pueraria lobata (Fabaceae), an introduced, clonal, invasive plant of the southeastern United States. American Journal of Botany, 87, 1240 - 1245 .

Ren MX, Zhang QG, Zhang DY (2005). Random amplified polymorphic DNA markers reveal low genetic variation and a single dominant genotype in Eichhornia crassipes populations throughout China. Weed Research, 45, 236-244.

Sakai AK, Allendorf FW, Holt JS, Lodge DM, Molofsky J, With KA, Baughman S, Cabin RJ, Cohen JE, Ellstrand NC, McCauley DE, O' Neil P, Parker IM, Thompson JN, Weller SG (2001) . The population biology of invasive species. Annual Review of Ecology and Systematics, 32, $305-332$.

Schierenbeck KA, Hamrick JL, Mack RN (1995). Comparison of allozyme variability in a native and an introduced species of Lonicera. Heredity, 75, $1-9$.

Shao H(邵华), Peng SL(彭少麟), Liu YX(刘运笑), Zhang C (张弛), Xiang YC(向言词) (2002). The biological control and the natural enemy of Mikania micrantha H.B.K.'s in China. Ecologic Science (生态科学), 21,33-36. (in Chinese with English abstract)

Shao WT(邵婉婷)，Han SC(韩诗畴)，Huang SS(黄寿山)，Liu WH(刘文惠), Li KH(李开煌), Peng TX (彭统序), Li LY (李丽英) (2002). Progress on the control of invasive weed Mikania micrantha. Guangdong Agricultural Sciences (广东农业 科学), 37(1), 43-45. (in Chinese)

Swamy PS, Ramakrishnan PS (1987a). Weed potential of Mikania micrantha H.B.K., and its control in fallows after shifting agriculture (Jhum) in North-East India. Agriculture, Ecosystems and
Environment, 18, 195 - 204

Swamy PS, Ramakrishnan PS (1987b) . Contribution of Mikania micrantha during secondary succession following slash-and-burn agriculture (Jhum) in North-East India. I. Biomass litterfall and productivity. Forest Ecology and Management, 22, 229 - 237.

Tsumura Y, Ohba K, Strauss SH (1996). Diversity and inheritance of inter-simple sequence repeat polymorphisms in Douglas fir (Pseudotsuga menzesii) and sugi (Cryptomeria japonica). Theoretical Application of Genetics, 92, 40-45.

Wain RP, Haller WT, Martin DF (1984). Genetic relationship among two forms of alligator weed. Journal of Aquatic Plant Management, 22, $104-105$.

Wang BS (王伯荪), Liao WB (廖文波), Miao RH (缪汝槐) (2001) . Revision of Mikania from China and the key of four relative species. Acta Scientiarum Naturalium Universitis Sunyatseni (中山大学学报 (自然科学版)), 40 (5), 72 - 75. (in Chinese with English abstract)

Wang BS (王伯荪), Wang YJ (王勇军), Liao WB (廖文波) (2004). Invasion Ecology and Control on Mikania micrantha (外 来杂草薇甘菊的入侵生态及其治理). Science Press, Beijing. (in Chinese)

Wei XY, Huang HJ, Wu P, Cao HL, Ye WH (2004). Phenolic constituents from Mikania micrantha. Biochemical Systematics and Ecology, 32, 1091 - 1096.

Wen DZ (温达志), Ye WH (叶万辉), Feng HL (冯惠玲), Cai CX (蔡楚雄) (2000). Comparison of basic photosynthetic characteristics between exotic invader weed Mikania micrantha and its companion species. Journal of Tropical and Subtropical Botany (热带亚热带植物学报)，8，139-146.（in Chinese with English abstract)

Wolfe AD, Xiang QY, Kephart SR (1998). Assessing hybridization in natural populations of Penstemon (Scrophulariaceae) using hypervariable intersimple sequence repeat (ISSR) bands. Molecular Ecology, 7, 1107 - 1125 .

Wright S (1978). Evolution and the Genetics of Populations Vol 4. Variability Within and Among Natural Populations. University of Chicago Press, Chicago.

Yang QH(杨期和), Feng HL(冯惠玲), Ye WH (叶万辉), Cao HL(曹洪麟)，Deng X(邓雄 $) ， \mathrm{Xu} \mathrm{KY}$ (许凯扬 $)$ （2003）。An investigation of the effects of environmental factors on the flowering and seed setting of Mikania micrantha H. B. K. (Compositae). Journal of Tropical and Subtropical Botany (热带亚热带植物学 报), 11, 123 - 126. (in Chinese with English abstract)

Ye WH, Li J, Cao HL, Ge XJ (2003) . Genetic uniformity of Alternanthera philoxeroides in South China. Weed Research, 43, 297 302. 
Ye WH, Mu HP, Cao HL, Ge XJ (2004). Genetic structure of the invasive Chromolaena odorata in China. Weed Research, 44, 129 -135 .

Yeh FC, Boyle TJB (1997). Population genetic analysis of co-dominant and dominant markers and quantitative traits. Belgian Journal of Botany, 129, 157.

Zan QJ(备启杰), Wang BS(王伯荪), Wang YJ(王勇军), Liao WB(廖文波), Li MG(李鸣光), Xu HL(徐华林) (2002). The ecological evaluation on the controlling Mikania micrantha by Cuscuta campestris. Acta Scientiarum Natralium Universitis Sunyatseni (中山大学学报 (自然科学版) ), 41 (6), 60-63. (in Chinese with English abstract)

Zan QJ(备启杰), Wang YJ(王勇军), Wang BS (王伯荪), Liao WB (廖文波), Li MG (李鸣光) (2000). The distribution and harm of the exotic weed Mikania micarantha. Chinese Journal of Ecology (生态学杂志), 19(6), 58-61. (in Chinese with English abstract)

Zhang FM(张富民), Ge S(葛颂) (2002). Data analysis in population genetics. I. Analysis of RAPD data with AMOVA. Biodiversity Science (生物多样性), 10,438-444.（in Chinese with
English abstract)

Zhang WY(张炜银), Wang BS(王伯荪), Li MG(李鸣光), Zan QJ(冬启杰), Wang YJ(王勇军) (2002a). The effects of light intensity on growth and morphology in Mikania micrntha seedlings. Sun Yatsen University Forum (中山大学学报论丛), 22(1), 222 - 226. (in Chinese with English abstract)

Zhang WY(张炜银), Wang BS(王伯荪), Liao WB(廖文波), Li $\operatorname{MG}$ (李鸣光), Wang YJ (王勇军), Zan QJ (各启杰) $(2002 b)$. Progress in studies on an exotic vicious weed Mikania micrantha. Chinese Journal of Applied Ecology (应用生态学 报), 13, 1684 - 1688. (in Chinese with English abstract)

Zhang WY(张炜银), Wang BS(王伯荪), Zhang JL(张军丽), Li MG (李鸣光), Zan QJ(各启杰), Wang YJ(王勇军) (2002c). Study on the structure and dynamics of seedlings of Mikania micrantha populations. Acta Scientiarum Naturalium Universitis Sunyatseni (中山大学学报 (自然科学版) ), 41(6), 64-66. (in Chinese with English abstract)

Zietkiewicz E, Rafalski A, Labuda D (1994). Genome fingerprinting by simple sequence repeat (SSR)-anhorcd polymerase chain reaction amplification. Genomics, 20, 176-183. 